\title{
Resect To Restore: Hemisection - A Case Report
}

DR. Vishwas Sangappa Madhudi, MDS Reader,

Dept. of. Conservative Dentistry and Endodontics, SJM.Dental College \&Hospital

Chitradurga.

Email: vishwas.madhudi@yahoo.com

Phone: $9880154640 /$ 080-23332795
Dr. Vasundhara Shivanna, MDS Proffessor \& Principal

Dept of Conservative Dentistry \& Endodontics, College of Dental Sciences, Davangere-577002

\section{ABSTRACT:}

Hemisection is a corrective periradicular surgery, carried usually in mandibular molars to preserve the maximum amount of supporting structures of the natural dentition in a state of health and function.

Hemisection is done in mandibular molars wherein one of the two roots is devoid of bone and periodontal support. The main intention of hemisection is to retain a part of tooth root while removing the deceased part, which has a poor prognosis.

In this article hemisection for a left mandibular molar with Grade I mobility and no migration of the affected tooth No.36, with exudation from the pocket is described and discussed as a treatment approach for a lesion of primary periodontal origin with secondary endodontic involvement, similar to observations made by Hamp et $\mathrm{al}^{19}$.

The indications advantages and limitations of hemisection as well as recommended literature on the subject are discussed.

Key words: Hemisection, Periradicular, granulomatous, Vertical cut method.

\section{INTRODUCTION:}

'Hemisection", meaning half-cut in Greek \& Latin is a corrective periradicular surgery, carried usually in mandibular molars wherein one of the two roots is devoid of bone and periodontal support. The main intention of hemisection is to retain a part of tooth root while removing the deceased part, which has a poor prognosis.

Farrar's proposition that, "saving part of a tooth is better than removing it".

G.V. Black stated that in dental practice a valuable tooth can be retained by amputation and removal of one of its deceased root.

In a multirooted tooth;

1. Severe bone loss around an isolated root threatening the health of other root.

2. Grade III and Grade IV furcation involvement. Hemisection can be one of the treatment modality.

3. Severe carious destruction of one of the segment of the tooth rendering it non restorable.

4. Endodontically untreatable roots because of mechanical inoperability or refractory situation.

\section{CASE REPORT:}

A 32 year old male patient reported to the Department of Conservative dentistry and Endodontics,
College of Dental Sciences, Davangere, with a chief complaint of pain while chewing in the left lower jaw.

On clinical examination there was no carious involvement of any tooth in that region. The oral hygiene status was poor with inflamed and recessed gingiva around the mesial root of 36 . On probing there was approximately $10 \mathrm{~mm}$ pocket depth only on the mesial side.

There was Grade I mobility and no migration of the affected tooth no.36, but it was involved with exudation form the pocket. There was no intraoral sinus. The vitality test of no.36 showed positive response.

Intraoral periapical (IOPA) radiographic examination revealed a complete $(100 \%)$ bone loss in relation to mesial root of 36 , extending to the furcation. However bone was intact around the distal root of 36 , the mesial and distal roots were spaced apart.(fig-1)

A treatment plan was generated taking into account the Endodontic and Prosthodontic 
considerations and treatment was executed hand in hand.

\section{MANAGEMENT:}

\section{First Appointment:}

Patient was posted for intentional root canal therapy, a day prior to surgery. Local anaesthesia was administered and an ideal access was opened in tooth 36 , following complete extirpation of the pulp, working

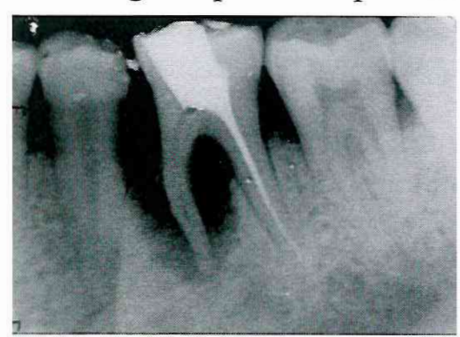

Fig 2 - Post obturation IOPA length was determined followed by biomechanical preparation and obturation in the distal canal only.(fig-2) Access cavity was filled with miracle mix.

\section{Second Appointment:}

On the day of surgery, the patient was prescribed analgesic 30 Minutes prior, to increase the pain threshold. LA was administered (2\% Lignocaine with $1: 80,000$ Adrenaline) in relation to tooth no.36, marginal gingiva was reflected around 36 with moons probe to expose the furcation, while taking care not to tear the tissue. From the buccal furcation a marking was placed extending onto the lingual furcation to help guide the bur path while hemisecting with the cut intended more on to the deceased part of tooth root and preserving as much as possible the distal part of tooth \& root.

With the help of a Micro motor hand piece and surgical length Tapering fissure diamond the tooth was sectioned into two halves along the marking placed earlier starting form buccal towards lingual. Continuous irrigation was carried out during cutting to

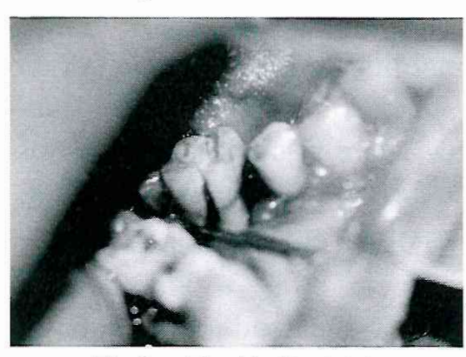

Fig 3 - The Vertical cut

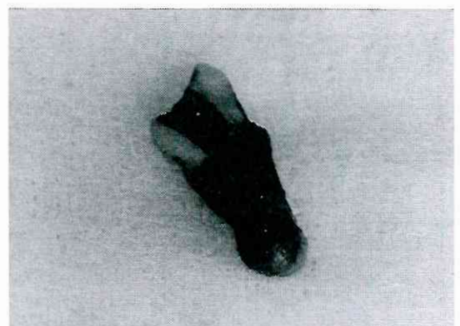

Fig 4 - Resected Mesial tooth-root with granulomatous tissue reduce the resecting temperatures near the bone. After confirming a Vertical cut separating both the halves (fig. 3 ) with the help of a periodontal probe. A lower premolar forceps was deeply engaged onto the mesial half of cut tooth 36 and extracted. The extracted mesial half of tooth-root of 36 showed large

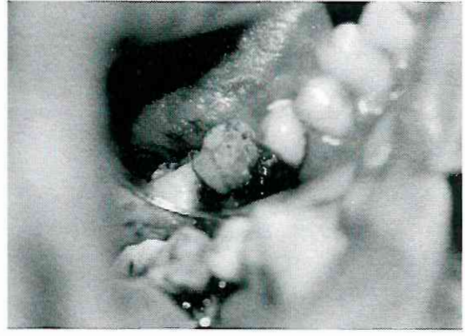

Fig 5 - Restored Distal retained tooth half with miracle mix

Fig 6 - Post operative IOPA

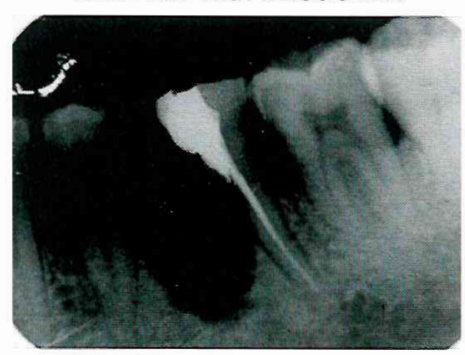

granulomatous tissue wrapped around the root extending up till the furcation (fig. 4). Radiograph was taken to ensure clean cut and any sharp projections on the distal half of tooth-root of 36 , the extracted socket was curetted and thoroughly irrigated after which the distal tooth root was contoured and the exposed tooth restored with miracle mix to a contour of a premolar (fig.5, 6).

Two interrupted sutures were placed after compressing the socket one distal to 35 and one mesial to 36 for a healthy gingival attachment. The distal half of the tooth was ground out of occlusion. Antibiotics and analgesics were prescribed, post-operative instructions were given and the patient was recalled after a week.

At the end of first week, the patient was examined and sutures were removed and the area was thoroughly irrigated. Patient was recalled at weekly intervals and at the end of 6-weeks post surgery the hemisected tooth was prepared along with tooth no. 35 as abutment and a provisional restoration was given with acrylic resin to maintain space and

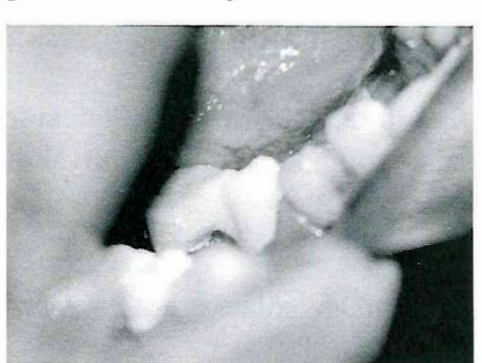

Fig 7 - Provisional Bridge restoration stabilize the remaining tooth $\mathrm{structures}$ (fig.7). Patient was recalled at $\mathrm{m}$ o $\mathrm{n} \mathrm{th} \mathrm{l} \mathrm{y}$ intervals and at the 3 months post-surgery, radiographic evaluation of bone fill of the socket was excellent.

A 3 unit fixed prosthesis was fabricated and cemented using 35 as mesial abutment. Later the case was clinically and radiographically evaluated after 6 months post surgery. The patient was fully satisfied by the functional aspect of the bridge.

\section{DISCUSSION:}

Increasing desire to preserve the tooth, "Hemisec- 
tion" procedure has gained a lot of value to retain the arch integrity. Even if one half of a tooth is saved it can provide sufficient support to adjacent members of the arch. Since hemisection is done in molar teeth which frequently serve as the posterior most abutment, retaining even half a tooth could avoid the need for removable prosthesis.

The mandibular first molars are more favourable than second molars, as the root of second molars are not as divergent as the first molars and the furcation is usually located at a more apical position on the root in relation to the crown of the tooth than on adjacent first molar teeth. 5

In the present case, the origin of the endo-perio problem was of primary periodontal origin with secondary endodontic involvement, similar to observations made by Hamp et $\mathrm{al}^{9}$. Hemisection can be done prior to or after hemisection procedure, long term success or failure seems to be unrelated to, which is done first, but majority researchers and clinicians agree that whenever possible, the endodontic treatment should be carried out first, as done in the present case too.

Advantage of Performing Endo treatment first is;

a) The pulp usually vital is uncontaminated and the endodontic treatment can be done in single visit if desired so.

b) Patient does not have pain and sensitivity after the hemisection.

c) Endodontist does not have to inject into a recently traumatized area which frequently is difficult to anesthetise and coupled with hyperemia or pulpitis may result in pain during endodontic treatment.

d) Isolation of remaining root without contamination is more difficult after hemisection.

e) Finally, the teeth with advanced furcation involvement often need endodontic therapy first because of presence of lateral or accessory canals in the furcation or in the interradicular areas.

The "vertical cut method" in Hemisection of mandibular molars as executed in the present case, is an excellent and time-tested procedure. If the furcation can not be located properly, as silver point inserted inside the furcation helps in making a cut. Always the section should be done at the cost of tooth part to be removed.

The successful use of hemisected tooth as bridge abutment in the present case report confirms the results of Nyman et al $(1975)^{4}$ and Erpensten $(1983) .^{2}$ Even teeth with markedly reduced periodontal support may be used as bridge abutments following periodontal treatment, maintenance of a high standard of plaque control and reconstruction of a stable occlusion.

\section{CONCLUSION :}

"We cannot change the wind....... But we can adjust the sail"

Today's success of preventive and conservative dentistry is to preserve the maximum amount of supporting structures of the natural dentition in a state of health and function.

A multidisciplinary approach has proven to be a valuable aid in obtaining this objective.

\section{REFERENCES :}

1. Abrams, A. and Trachtenberg, DI: Hemisection technique and restoration. Dent Clin North Am.1974; 18: 415-444.

2. Erpenstein, H.: A 3-year study of hemisected molars. J Clin Periodontal.1983; 10: 110.

3. Green, L.N.: Hemisection and root amputation. J Am Dent Asso.1986; 112: 511-518.

4. Nyman, S., Lindhe, J., Lundgren, D.: The role of occlusion for the of fixed bridges in patients with reduced periodontal tissue support. J Clin Periodontal.1975; 2: 53-66

5. Goldman. Hand Cohen.W: Periodontal therapy 6th edition p-1026-1027, St lows,cv Mosby co 1980.

6. Endodontics.5th edition. John I .Ingle.p-669-741.

7. Contemporary Fixed Prosthodontics, 3rd edition, Rosensteil,p-123-125.

8. Bergenholtz.A.; Radisectomy of multirooted teeth.JADA 1972:85;870-875.

9. Hamp SE, Nyman s, Lindhe J; Periodontal treatment of multirooted teeth-results after 3 years. J.clin Periodont.1975:2;126-135. 